\title{
A Rare Case of Trapped Lung Cause by Chronic Pleural Effusion: Another Etiology of This Uncommon Disease
}

\author{
Hardik Fichadiya ${ }^{1 *}$, Tanya Shankar ${ }^{1}$, Sherif Elkattawy ${ }^{1}$, Harshil Fichadiya ${ }^{2}$, Aditya Patel ${ }^{3}$, Ruhma Ali $^{3}$ and \\ Michael Brescia ${ }^{4}$ \\ ${ }^{1}$ Internal Medicine, Rutgers-New Jersey Medical School/Trinitas Regional Medical Center, USA \\ ${ }^{2}$ Department of Internal medicine, Monmouth Medical Center, USA \\ ${ }^{3}$ Department of Internal medicine, St. Michaels Medical Center, USA \\ ${ }^{4}$ Department of Pulmonology and Critical Care, Trinitas Regional Medical Center, USA \\ Submission: December 16, 2021; Published: January 18, 2022 \\ *Corresponding author: Hardik Fichadiya, Internal Medicine, Rutgers-New Jersey Medical School/Trinitas Regional Medical Center, Elizabeth, \\ 1070 Morris Ave, Apt 1123, Union, NJ 07083, USA
}

\section{Abstract}

Trapped lung is defined by the inability of the lung to expand and fill the thoracic cavity. It is an outcome of fibrosis and thickening of visceral pleural preventing lung re-expansion. We present a 62 -year-old women with past medical history of chronic kidney disease and heart failure with preserved ejection fraction who presented with bilateral pleural effusions. Effusion on the right side was tapped draining a transudative fluid. Chest X ray on the following day showed a pneumothorax at the right lung base, the margins of which coincided which that of the effusion. The following day she now developed a hydro-pneumothorax in the same location. One rare yet reported cause of trapped lung is chronic pleural effusions in the setting of decompensated heart failure.

Keywords: Trapped lung; Pneumothorax; Congestive heart failure; Pleurodesis; Chronic pleural effusions

\section{Introduction}

Trapped lung is defined by the inability of the lung to expand and fill the thoracic cavity. It is an outcome of fibrinous or granulomatous pleuritis. It is historically recognized as a complication of therapeutic pneumothorax for treatment of tuberculosis. However, today we know that it is a consequence of numerous processes (e.g cardiac surgery, chest trauma, inadequately treated parapneumonic effusion, pleural effusions) involving the pleura [1]. One rare yet reported cause of trapped lung is chronic pleural effusions in setting of decompensated heart failure. We present a case of a 62 year old female with history of congestive heart failure with preserved ejection fraction who was found to have trapped lung in setting of chronic pleural effusions.

\section{Case Report}

A 62 year old Hispanic women with a past medical history of stage 4 chronic kidney disease on hemodialysis, congestive heart failure with preserved ejection fraction, diabetes mellitus, hypertension, chronic obstructive pulmonary disease and seizure disorder became unresponsive 1.5 hours into her dialysis session during which 150 milliliters of fluid was removed. She was hemodynamically stable before the procedure and was at her baseline mental status where she is slight confused and oriented to only place and person. On presentation she was comatose with a Glasgow Coma Scale score of 3 ( 1 for eye movement, 1 for motor and 1 for verbal). Vitals were blood pressure: 103/56mmHg, heart rate: 70/min, temperature 37C (Rectal) and respiratory rate $25 /$ min (with rapid shallow breaths).

Lung exam showed bibasilar crackles with occasional wheezes. arterial blood gas was consistent with hypercapnia respiratory failure with $\mathrm{pH}: 7.13, \mathrm{PCO}_{2}: 81, \mathrm{HCO}_{3}: 23.1, \mathrm{SpO}_{2}: 98.8$ and $\mathrm{PO}_{2}: 164$. Labs were significant Blood urea nitrogen: $22 \mathrm{mg} /$ $\mathrm{dl}$, creatinine: $5.2 \mathrm{mg} / \mathrm{dl}$, estimated glomerular filtration rate: $9 \mathrm{ml} / \mathrm{min}$, microcytic anemia with hemoglobin: $6.5 \mathrm{~g} / \mathrm{dl}$, white blood cells: $3700 /$ cubic millimeter and Platelet: 104,000/cubic millimeter. Electrocardiogram was within normal limits. CT Head was unremarkable except for old lacunar infarcts in the pons. The patient was intubated and transferred to the intensive care unit on ventilator settings of tidal volume: 400 , respiratory rate: 16 , Peak end expiration pressure: 5 and fraction of inspired oxygen: 40. Chest X ray and CT showed bilateral pleural effusions (right 
greater than left) and underlying consolidation of the right basilar segments (Figure 1).

Echocardiogram from 1 month ago showed finding consistent with hypertensive heart disease and heart failure with preserved ejection fraction (LVEF: 55-60\%). Severe left atrial dilation was noted with moderate mitral regurgitation, moderate pulmonary regurgitation, and mild elevation of pulmonary systolic artery pressure. The patient got hemodialysis the next day and her hypertensive heart disease was treated with Isosorbide mononitrate, amlodipine, carvedilol and lisinopril. Her CXR following hemodialysis showed slight reduction of the effusion on the right (Figure 2). She did not require vasopressor support in the ICU and was then transferred to the medical floors on two liters nasal cannula. The following day right sided thoracocentesis was performed draining 1.4 liters of cloudy transudative fluid.

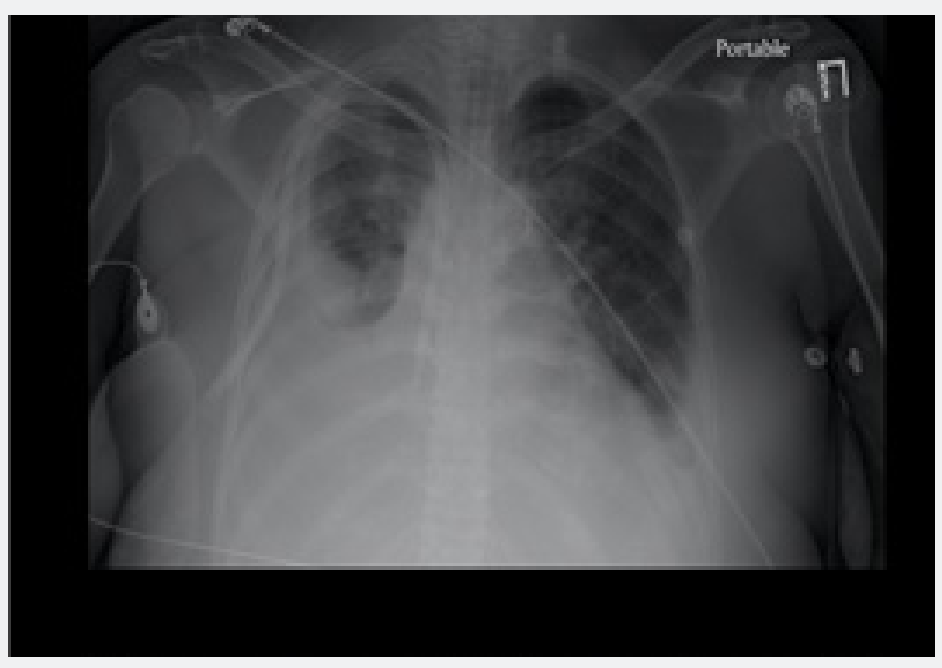

Figure 1:

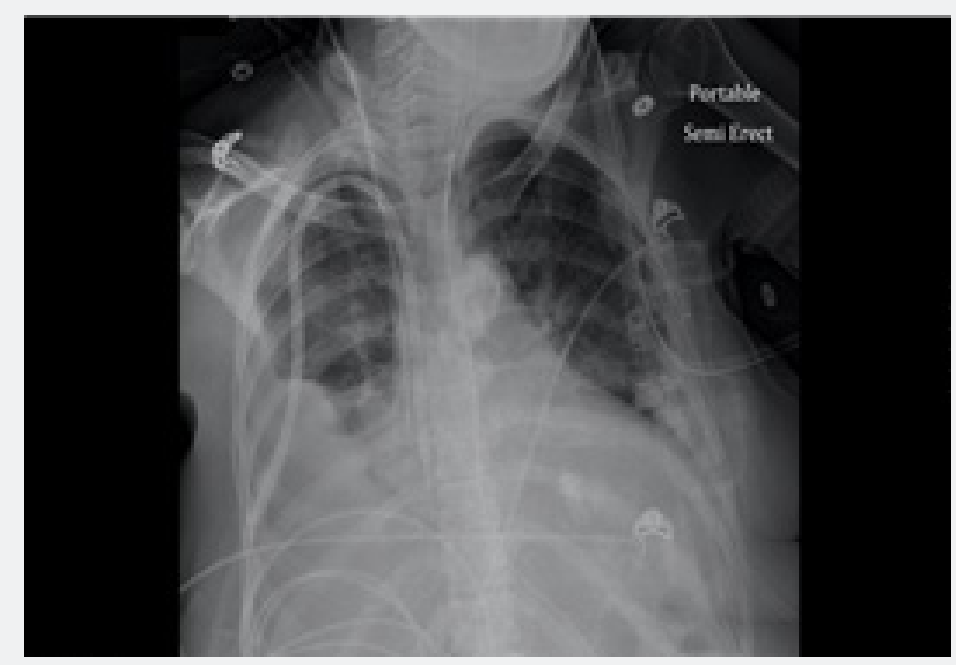

Figure 2:

Pleural fluid analysis was significant for 5000 red blood cells, 116 white blood cells (27\% Polymorphs, 59\% lymphocytes, 6\% monocytes, 5\% mesothelial cells and 3\% macrophages.), glucose $177 \mathrm{mg} / \mathrm{dl}$, pleural fluid protein: $1.8 \mathrm{~g} / \mathrm{dl}$ with pleural fluid/serum protein ratio: 0.38 and pleural fluid Lactate Dehydrogenase (LDH):77 U/L with pleural fluid Lactate Dehydrogenase/serum Lactate Dehydrogenase ratio 0.43 . These findings are consistent with transudative effusion. Following the procedure chest $\mathrm{X}$ ray showed pneumothorax like picture on the right lung base with its location and margin coinciding with that of the pleural effusion before thoracocentesis. At this time the patient's vitals were stable, she was in no distress, was speaking complete sentences and saturating $100 \%$ on room air. Her effusion in the left pleural space is unchanged from that of the previous $\mathrm{X}$ ray (Figure 3). 
$\mathrm{X}$ ray on the following was now significant for a right hydropneumothoraxin the samelocation. Effusion the leftstill unchanged from that on the left (Figure 4). The patient continued to be at her baseline health status and had no active complains from the effusions. As the patient remained stable, she was discharged to the nursing home with regular outpatient hemodialysis sessions and aggressive treatment of her hypertensive heart disease with carvedilol, amlodipine, hydralazine and lisinopril (Figure 5).

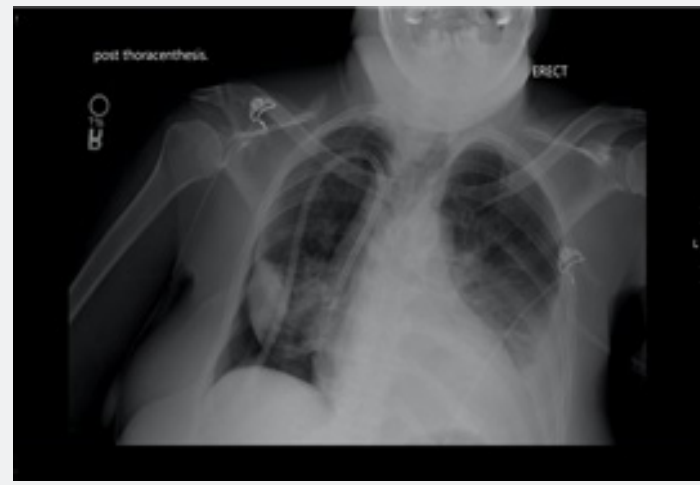

Figure 3:

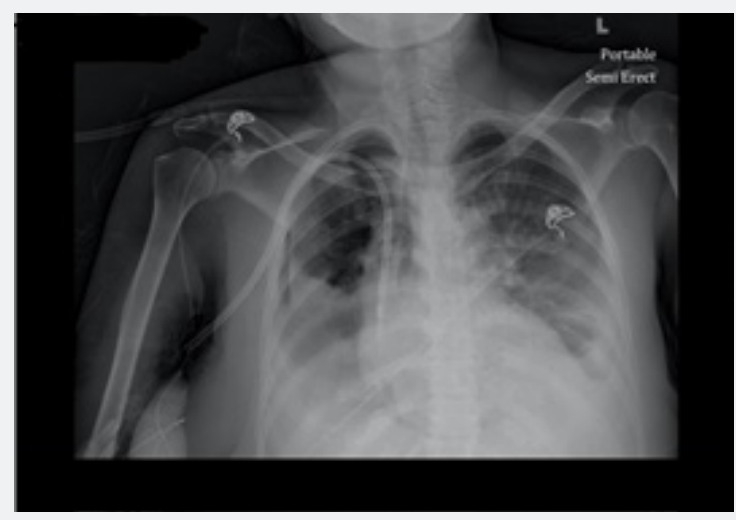

Figure 4:

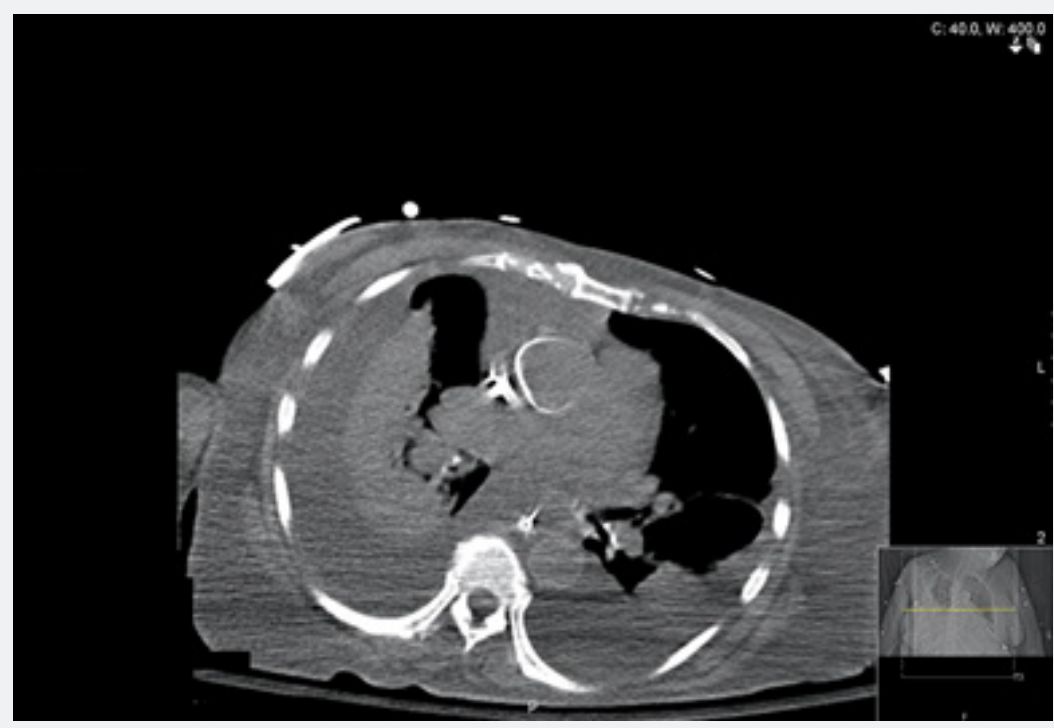

Figure 5: $\mathrm{X}$ ray showing right sided trapped lung as explained in the text above. 


\section{Discussion}

Hypertension is a leading cause of cardiomyopathy. In hypertensive heart disease, left ventricular hypertrophy due to increased afterload leads to decreased end diastolic pressure and increased pulmonary capillary wedge pressure. This leads to increased end capillary hydrostatic pressure and hence leakage of fluid into the lung interstitium. Fluid then travels across the visceral pleura into the pleural space. This is how CHF is a common cause of bilateral pleural effusions [2]. Chronic pleural effusion is a common cause of trapped lung. Fluid in the pleural space restricts expansion of the lung tissue. Lung is able to fully expand when the fluid is removed by thoracentesis. However, over time the cortex of the visceral pleura undergoes fibrosis and thickens, preventing re expansion of the lung even after thoracentesis [3].

The duration of disease course is unknown as in most cases patients remain asymptomatic, so the condition goes unnoticed [4]. Trapped lung essentially develops when the lung is unable to re expand and the pleural cavity fills up with fluid and air, creating a hydropneumothorax. Inflammatory insult caused by pneumonia or hemothorax, pleural malignancy, spontaneous pneumothorax, coronary artery bypass surgery, uremia and rheumatoid pleuritic can be attributed as the causes [5]. Symptoms are similar to those of pneumothorax, caused due to ventilation-perfusion mismatch, consisting of shortness of breath, absence of breath sounds, hyperresonance to percussion, hypoxemia [3].

Chest radiography post initial thoracentesis would show thickened visceral pleural cortex. CT scan would also be helpful in visualizing the thickened pleura and trapped lung tissue, but it may be falsely thickened due to overlying exudate and debris [6]. Analysis of the pleural fluid should be done to rule out malignant effusion, so treatment can be directed towards symptomatic relief as it has a short-expected survival [7]. Definitive treatment is surgical decortication but is reserved for patients with symptoms and who are able to tolerate. Bronchial obstruction is ruled out before surgical intervention is planned [6].

In this case, pleural effusion was the cause for formation of trapped lung. Imaging post thoracentesis did not show resolution of the effusion. Following imaging showed formation of hydropneumothorax in the same location. Patient was not a surgical candidate due to age. She was only managed medically for her diastolic heart failure and through ultrafiltration to prevent re accumulation of fluid.

\section{References}

1. Doelken P, Sahn SA (2001) Trapped lung. Semin Respir Crit Care Med 22(6): 631-636.

2. Porcel JM (2010) Pleural effusions from congestive heart failure. Semin Respir Crit Care Med 31(6): 689-697.

3. Albores J, Wang $\mathrm{T}$ (2015) Images in clinical medicine. Trapped lung. $\mathrm{N}$ Engl J Med 372: e25.

4. Pereyra MF, Ferreiro L, Valdes L (2013) Unexpandable lung. Arch Bronconeumol 49(2): 63-69.

5. Kollef MH (1990) Trapped-lung syndrome after cardiac surgery: a potentially preventable complication of pleural injury. Heart Lung 19(6): 671-675.

6. Rathinam S, Waller DA (2013) Pleurectomy decortication in the treatment of the "trapped lung" in benign and malignant pleural effusions. Thorac Surg Clin 23(1): 51-61.

7. Doelken P (2008) Clinical implications of unexpandable lung due to pleural disease. Am J Med Sci 335(1): 21-5.

\section{Your next submission with Juniper Publishers} will reach you the below assets

- Quality Editorial service

- Swift Peer Review

- Reprints availability

- E-prints Service

- Manuscript Podcast for convenient understanding

- Global attainment for your research

- Manuscript accessibility in different formats ( Pdf, E-pub, Full Text, Audio)

- Unceasing customer service

Track the below URL for one-step submission https://juniperpublishers.com/online-submission.php 